\title{
Constraints on radiative dark-matter decay from the cosmic microwave background
}

\author{
Le Zhang, ${ }^{1,2, *}$ Xuelei Chen, ${ }^{1, \dagger}$ Marc Kamionkowski, ${ }^{3, \$}$ Zong-guo $\mathrm{Si}^{2,8}$ and Zheng Zheng ${ }^{4, \|}$ \\ ${ }^{1}$ National Astronomical Observatories, Chinese Academy of Sciences, Beijing, 100012, China \\ ${ }^{2}$ Department of Physics, Shandong University, Jinan, 250100, China \\ ${ }^{3}$ California Institute of Technology, Mail Code 130-33, Pasadena, California 91125, USA \\ ${ }^{4}$ Institute for Advanced Study, Einstein Drive, Princeton, New Jersey 08540, USA
}

(Received 18 April 2007; published 18 September 2007)

\begin{abstract}
If dark matter decays to electromagnetically interacting particles, it can inject energy into the baryonic gas and thus affect the processes of recombination and reionization. This leaves an imprint on the cosmic microwave background (CMB): the large-scale polarization is enhanced, and the small-scale temperature fluctuation is damped. We use the Wilkinson Microwave Anisotropy Probe (WMAP) three-year data combined with galaxy surveys to constrain radiatively decaying dark matter. Our new limits to the darkmatter decay width are about 10 times stronger than previous limits. For dark-matter lifetimes that exceed the age of the Universe, a limit of $\zeta \Gamma_{\chi}<1.7 \times 10^{-25} \mathrm{~s}^{-1}$ (95\% C.L.) is derived, where $\zeta$ is the efficiency of converting decay energy into ionization energy. Limits for lifetimes short compared with the age of the Universe are also derived. We forecast improvements expected from the Planck satellite.
\end{abstract}

DOI: 10.1103/PhysRevD.76.061301

PACS numbers: 98.70.Vc, 95.35.+d

\section{INTRODUCTION}

After decades of research, the nature of the dark matter remains elusive. Nevertheless, many properties of dark matter can be inferred from analysis of astrophysical processes that it might affect. In particular, if the dark-matter (DM) particle is unstable, or if it is produced by decay of an unstable progenitor, then energy released during the decay could affect recombination and reionization. This scenario received much interest $[1,2]$ after the release of the firstyear Wilkinson Microwave Anisotropy Probe (WMAP) results [3], which showed a large temperature-polarization (TE) cross correlation, indicating an earlier epoch of reionization than is easily accommodated with the $\Lambda \mathrm{CDM}$ model [4]. In this scenario, the decay of the DM particle supplies enough energy to provide early reionization.

Recently, the WMAP team has released data from three years of observation [5], and the implications for reionization are now more in line with the conventional reionization scenario, though with a lowered matter density $\Omega_{m}$ and power-spectrum normalization, the demand on early and efficient star formation still exists [6]. While the original motivation of explaining reionization with DM decay is now less compelling, the calculations of the effects of DM decay on the CMB can be turned now, with the new data, to deriving more stringent constraints to decaying DM [7].

Although 21-cm observations may some day be employed to probe particle decay during the dark ages [8], the best present constraints come from the CMB. The CMB is affected by the ionization history in several ways: at

\footnotetext{
*zl@cosmology.bao.ac.cn

xuelei@cosmology.bao.ac.cn

*kamion@tapir.caltech.edu

$\S$ zgsi@sdu.edu.cn

" zhengz@ias.edu
}

large scales, Thomson scattering by free electrons after reionization generates polarization from temperature anisotropies. This shows up as an enhanced TE cross correlation and polarization autocorrelation (EE). This is why the new WMAP data, with greatly improved measurements of large-angle polarization, will provide much more stringent constraints to radiatively decaying DM. At smaller scales, Thomson scattering damps the primordial temperature anisotropy by a factor of $e^{-2 \tau}$. If the matter power spectrum can be measured independently by other means, e.g., with large-scale-structure (LSS) surveys, then this effect can also be used to constrain the ionization history.

In the present work, we use the currently available CMB data, including WMAP [5], ACBAR [9], Boomerang [10], CBI [11], and VSA [12], and the SDSS [13] and 2dF [14] galaxy surveys, to constrain radiatively decaying DM. This work updates, expands, and improves upon our previous work [1] in several ways. In addition to using the new WMAP data, which is now far better suited for this analysis than the first-year data, we also include LSS data which, as we shall see, provides some small additional improvement. We have also improved our methods, using now Markov Chain Monte Carlo (MCMC) techniques for parameter fitting, as we have done for analogous constraints to DM annihilation in Ref. [15]. This allows a better understanding of the degeneracy between inferred parameters, and it also provides more reliable error estimates. This work also differs from Ref. [16] by searching a broader range parameter space, doing a full likelihood analysis, and including LSS. In the regions where our parameter spaces overlap, our limit is a factor of a few better than theirs. References [17,18] also overlap with this work, but the former studies only several particular DM candidates, rather than surveying the entire decaying-DM parameter space, while the latter considers heating/ionization only at recombination. 
Our calculation of the effects of DM decay on recombination, reionization, and heating of the intergalactic medium, as well as their subsequent impact on the CMB temperature and polarization power spectra follows that in Ref. [1]. The ionization rate from DM decay is

$$
I_{\chi}=\chi_{i} f\left(f_{\chi} \Omega_{\chi} / \Omega_{b}\right)\left(m_{b} c^{2} / E_{b}\right) \Gamma_{\chi} e^{-\Gamma_{\chi} t},
$$

where $\Gamma_{\chi}$ is the decay width (inverse lifetime) of the decaying particle, $m_{b}$ is the mass of the baryon particle (hydrogen), $\left(\Omega_{\chi} / \Omega_{b}\right)$ is the initial ratio of DM mass to baryonic mass, $f$ is the fraction of the decay energy deposited in the baryonic gas, $c$ is the speed of light, $f_{\chi}=$ $\Delta E /\left(m_{\chi} c^{2}\right), \Delta E$ is the energy released in the decay, $E_{b}=$ $13.6 \mathrm{eV}$ is the binding energy of hydrogen, and $t$ is the cosmological time. The rate at which DM decay contributes to the heating of the intergalactic medium (IGM) is

$$
K_{\chi}=\chi_{h} f\left(f_{\chi} \Omega_{\chi} / \Omega_{b}\right) m_{b} c^{2} \Gamma_{\chi} e^{-\Gamma_{\chi} t} .
$$

The decay energy deposited in the gas contributes both to the ionization and heating of the gas. We model the division between these by an ionizing fraction $\chi_{i}$ and heating fraction $\chi_{h}$ given by [1]

$$
\chi_{i}=\left(1-x_{e}\right) / 3, \quad \chi_{h}=\left(1+2 x_{e}\right) / 3,
$$

where $x_{e}$ is the ionization fraction. The effects of decay on recombination can be determined completely by two independent parameters, $\Gamma_{\chi}$ and $\zeta$, where $\zeta=f f_{\chi}$, and is related to the quantity $\xi$ defined in Ref. [1] by $\xi=\Gamma_{\chi} \zeta$.

We have modified the recombination code RECFAST [19], which is used in the CMB code CAMB [20] for the calculation of the ionization history, to account for these extra contributions to ionization and heating. The CAMB code is used by the MCMC code COSMOMC [21] as its CMB driver. We also modified COSMOMC so we could vary the new parameters $\Gamma_{\chi}$ and $\zeta$ along with the cosmological parameters. For the present study, we have considered the following set of 6 cosmological parameters: $\left\{\Omega_{b} h^{2}, \Omega_{c} h^{2}, \theta, \tau, n_{s}, A_{s}\right\}, n_{s}$ and $A_{s}$ are the spectral index and amplitude of primordial perturbations, and $\tau$ is the optical depth in the absence of DM decay. When we include LSS (which also encoded baryon acoustic oscillation information) in our analysis, we also marginalize over the galaxy bias $b$, and use data with $k<0.2 \mathrm{Mpc} / h$ where the growth is linear.

When the ionization history is altered, the TE and EE spectra are enhanced at large scales (small $\ell$ ). The TT spectrum at large $\ell$ is damped by a factor of $e^{-2 \tau_{\text {tot }}}$, where $\tau_{\text {tot }}$ is the total optical depth. If the power-spectrum amplitude is not known a priori, the large- $\ell$ suppression may appear as a low- $\ell$ increase. However, if the matter power spectrum is obtained from galaxy surveys, the degeneracy between $\tau_{\text {tot }}$ and $A_{s}$ can be broken. Here we use the LSS power spectrum obtained in the SDSS [13] and 2dF [14] surveys. We will see, though, that quantitatively their inclusion improves the decaying-DM constraint only a little, as the absolute amplitude is not known in this data set, and the constraint comes only from the shape of the power spectrum.

If the decay lifetime is long compared with the age of the Universe, the exponential factor in Eqs. (1) and (2) reduce to unity, so we can parametrize the effects of DM decay fully by the combination $\zeta \Gamma_{\chi}$. In Fig. 1, we plot the marginalized probability distribution function (PDF) of $\zeta \Gamma_{\chi}$ and the relative mean likelihood, for both the constraint obtained from the CMB only and also that obtained by including also LSS. We find that these curves peak at $\zeta \Gamma_{\chi}=0$, indicating that the $\mathrm{CMB}$ is consistent with no significant DM decay. The PDF drops rapidly at $\zeta \Gamma_{\chi} \sim$ $10^{-25} \mathrm{~s}^{-1}$, and the $95 \%$ limit is at

$$
\zeta \Gamma_{\chi}<2.4 \times 10^{-25} \mathrm{~s}^{-1},
$$

from the CMB only. The constraint is improved slightly with the addition of LSS data; the $95 \%$ limit is then

$$
\zeta \Gamma_{\chi}<1.7 \times 10^{-25} \mathrm{~s}^{-1}
$$

in this case. The degeneracy of the bias with the galaxyclustering amplitude limits the power of including LSS. If the bias can be fixed by some other measurement, such as the three-point correlation function, then there should be further improvements better than those we have obtained here. For the present data, if we assume $\zeta \leq 0.1$, we can exclude models with lifetimes $\Gamma_{\chi}^{-1}<5.9 \times 10^{23} \mathrm{sec}$ in the long-lived case. This improvement over previous result [1] may provide strong constraint on new decaying-DM models such as those given in Ref. [22].

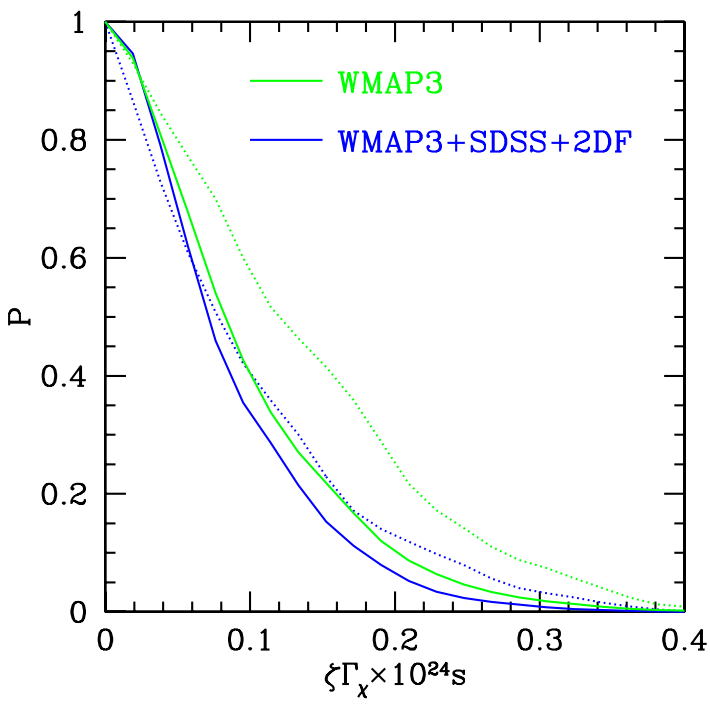

FIG. 1 (color online). The marginalized probability distribution function (solid curve) and the relative mean likelihood (dashed curve) of the $\zeta \Gamma_{\chi}$ parameter in the case of long lifetime, for the CMB-only (WMAP 3 yr) constraint (green upper curves), and the CMB and LSS (SDSS $+2 \mathrm{dF}$ ) constraint (blue lower curves). The normalization is such that the maximum of the function is 1 . 
For a decay lifetime shorter than the age of the Universe at the epoch of reionization $\left(\sim 10^{16} \mathrm{sec}\right)$, we need to fit the two parameters $\zeta$ and $\Gamma_{\chi}$ independently. In Fig. 2, we plot the excluded region $(2 \sigma)$ in the two-dimensional parameter space $\left(\log _{10} \zeta, \log _{10} \Gamma_{\chi}\right)$, obtained after marginalization over the other parameters. The middle filled region (red) is excluded by the current CMB and LSS data set. The boundary of this region is basically a straight line with $\zeta \Gamma_{\chi} \sim$ const. The line curves a little bit at the short-lifetime end $\left(\Gamma_{\chi} \sim 10^{-13} \mathrm{sec}\right)$. A fit to the boundary curve is given by

$y=\left\{\begin{array}{l}6.77+3.96275 x+0.25858 x^{2}+0.00445 x^{3}(x>-17), \\ -24.75-x(x<-17),\end{array}\right.$

where $x=\log _{10} \Gamma_{\chi}$ and $y=\log _{10} \zeta$. We have restricted the parameter space to $\Gamma_{\chi}<10^{-13} \mathrm{~s}^{-1}$, because for still shorter lifetime, much of the decay would happen before recombination and would thus not affect the ionization history of the Universe.

We will soon have CMB data of still better quality from the Planck satellite [23], to be launched soon. Anticipating this development, we use the Fisher-matrix formalism $[15,24]$ to forecast the future measurement error. We assume a sky coverage of 0.65 and adopt a fiducial model that best fits the WMAP three-year data. Because Planck will determine the B-mode polarization, we evaluate the constraint derived from the TT, TE, EE, and BB spectra, and use the covariance matrices of Ref. [25]. The resulting constraint is also plotted in Fig. 2, and a fit to it is given by

$$
y=\left\{\begin{array}{l}
3.6+2.645 x+0.114 x^{2}(x>-17), \\
-25.531-x(x<-17),
\end{array}\right.
$$

where $x=\log _{10} \Gamma_{\chi}$ and $y=\log _{10} \zeta$.

Dark-matter decay may also affect the global fitting of cosmological parameters. We expect the decay parameter

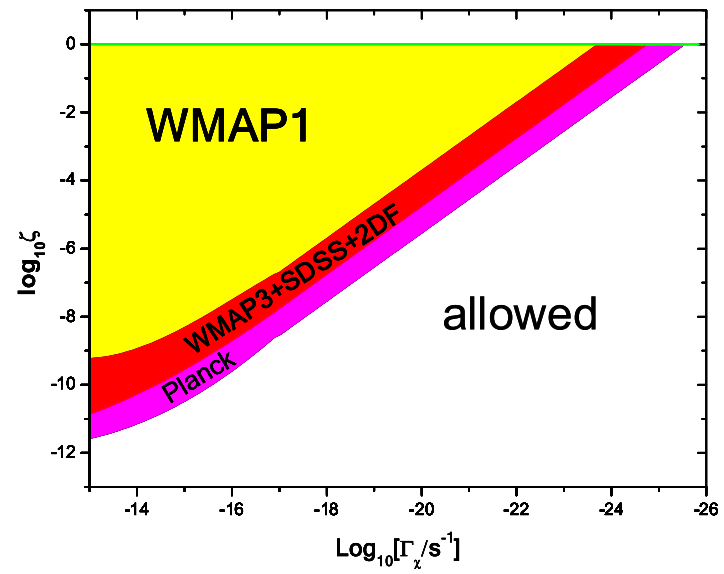

FIG. 2 (color online). The $2 \sigma$ constraints on the decaying-DM parameter space. We show the limits from the WMAP1 analysis of Ref. [1], our new constraint from WMAP3 + SDSS + 2dF, as well as our forecast for the reach of Planck. $\zeta \Gamma_{\chi}$ to correlate with optical depth $\tau$, as both of these are related to the ionization history. We also expect it to correlate with $A_{s}$ and $n_{s}$, as scattering from electrons damps small-scale CMB anisotropy. There should also be some correlation with $\Omega_{c}$, as this fixes the rate of input of decay energy. We plot the $2 \mathrm{D}$ contours of the $\zeta \Gamma_{\chi}$ parameter with other cosmological parameters in Fig. 3. There is indeed some correlation with the above parameters. By contrast, if we consider additional ionization and heating due to DM annihilation [15] (rather than decay), the correlation is very weak because DM annihilation occurs mainly during the earlier epoch of recombination when the DM density is higher [15]. However, the correlation is perhaps not quite as strong as one might have anticipated from the Fisher-matrix analysis [1]. This, we believe, is because the plotted contours follow marginalization, during which the correlation is weakened somewhat.

We have studied the impact of decaying DM on the ionization history and its subsequent effects on the CMB. Our investigation is largely model independent; i.e., it does not depend on the particular type of particle, but only on the decay width $\Gamma_{\chi}$ and energy-conversion efficiency $\zeta$. Dark-matter decay may affect the temperature and ionization fraction of the baryonic gas. It may also help produce or destroy molecular hydrogen, which plays an important role in the formation of the first stars [26]. We have not
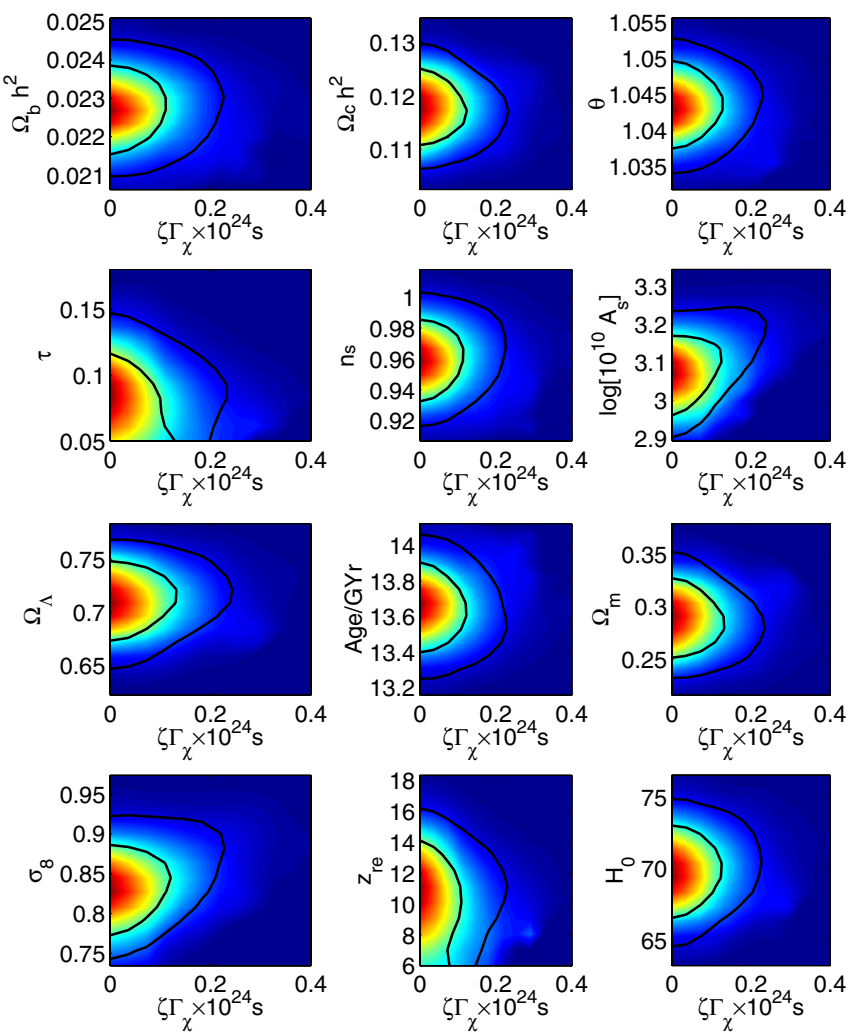

FIG. 3 (color online). The 2D contours of the distribution of $\zeta \Gamma_{\chi}$ and the background parameters for WMAP3 + SDSS + $2 \mathrm{dF}$ data. The color is for the relative mean likelihood. 
considered such effects in this work, but treated DM decay and star formation as two independent processes. We obtained constraints on DM decay by using data from WMAP's three-year results and the SDSS and 2dF galaxy surveys. We have derived limits on decay parameters [cf., Eqs. (4) and (6)]. The constraint on decaying DM will be further improved with future CMB experiments such as the Planck satellite [cf., Eq. (7)], and/or direct observation of the dark ages with 21-cm surveys [8].

\section{ACKNOWLEDGMENTS}

Our MCMC chain computation was performed on the Supercomputing Center of the Chinese Academy of
Sciences and the Shanghai Supercomputing Center. This work is supported by the National Science Foundation of China under the Distinguished Young Scholar Grant No. 10525314, the Key Project Grant No. 10533010, the 973 Program No. 2007CB815401, and by the Chinese Academy of Sciences under Grant No. KJCX3-SYW-N2. M. K. was supported by DOE No. DE-FG03-92-ER40701, NASA NNG05GF69G, and the Gordon and Betty Moore Foundation. Z.Z. was supported by NASA through HF01181.01-A from the Space Telescope Science Institute, which is operated by the Association of Universities for Research in Astronomy, Inc., for NASA under Contract No. NAS 5-26555.
[1] X. L. Chen and M. Kamionkowski, Phys. Rev. D 70, 043502 (2004).

[2] A. G. Doroshkevich et al., Astrophys. J. 586, 709 (2003); R. Bean, A. Melchiorri, and J. Silk, Phys. Rev. D 68, 083501 (2003); S. H. Hansen and Z. Haiman, Astrophys. J. 600, 26 (2004); E. Pierpaoli, Phys. Rev. Lett. 92, 031301 (2004); S. Kasuya, M. Kawasaki, and N. Sugiyama, Phys. Rev. D 69, 023512 (2004); S. Kasuya and M. Kawasaki, Phys. Rev. D 70, 103519 (2004); N. Padmanabhan and D. P. Finkbeiner, Phys. Rev. D 72, 023508 (2005).

[3] C. L. Bennett et al. (WMAP Collaboration), Astrophys. J. Suppl. Ser. 148, 1 (2003).

[4] Z. Haiman and G. P. Holder, Astrophys. J. 595, 1 (2003); M. Fukugita and M. Kawasaki, Mon. Not. R. Astron. Soc. 343, L25 (2003); N. Yoshida et al., Astrophys. J. 592, 645 (2003); A. Sokasian et al., Mon. Not. R. Astron. Soc. 344, 607 (2003).

[5] D. N. Spergel et al. (WMAP Collaboration), arXiv:astro$\mathrm{ph} / 0603449$;

[6] T. R. Choudhury and A. Ferrara, Mon. Not. R. Astron. Soc. 371, L55 (2006).

[7] M. Kamionkowski and A. Kosowsky, Annu. Rev. Nucl. Part. Sci. 49, 77 (1999).

[8] S. Furlanetto, S. P. Oh, and E. Pierpaoli, Phys. Rev. D 74, 103502 (2006); M. Valdes, A. Ferrara, M. Mapelli, and E. Ripamonti, Mon. Not. R. Astron. Soc. 377, 245 (2007).

[9] C. 1. Kuo et al. (ACBAR Collaboration), Astrophys. J. 600, 32 (2004).

[10] T.E. Montroy et al. (BOOMERanG Collaboration), Astrophys. J. 647, 813 (2006).

[11] T. J. Pearson et al. (CBI Collaboration), Astrophys. J. 591, 556 (2003).
[12] P.F. Scott et al. (VSA Collaboration), Mon. Not. R. Astron. Soc. 341, 1076 (2003).

[13] M. Tegmark et al. (SDSS Collaboration), Phys. Rev. D 74, 123507 (2006).

[14] S. Cole et al. (2dFGRS Collaboration), Mon. Not. R. Astron. Soc. 362, 505 (2005).

[15] L. Zhang, et al., Phys. Rev. D 74, 103519 (2006).

[16] S. Kasuya and M. Kawasaki, J. Cosmol. Astropart. Phys. 02 (2007) 010.

[17] M. Mapelli, A. Ferrara, and E. Pierpaoli, Mon. Not. R. Astron. Soc. 369, 1719 (2006).

[18] R. Bean, A. Melchiorri, and J. Silk, Phys. Rev. D 75, 063505 (2007);

[19] S. Seager, D. D. Sasselov, and D. Scott, Astrophys. J. Lett. 523, L1 (1999).

[20] A. Challinor and A. Lasenby, Astrophys. J. 513, 1 (1999); A. Lewis, A. Challinor, and A. Lasenby, Astrophys. J. 538, 473 (2000); http://camb.info.

[21] A. Lewis and S. Bridle, Phys. Rev. D 66, 103511 (2002); http://cosmologist.info/cosmomc; A. Lewis, J. Weller, and R. Battye, Mon. Not. R. Astron. Soc. 373, 561 (2006).

[22] J.A. R. Cembranos, J.L. Feng, and L.E. Strigari, arXiv:0704.1658.

[23] Planck Collaboration, Report No. ESA-SCI 2005; http:// www.rssd.esa.int/Planck.

[24] G. Jungman et al., Phys. Rev. Lett. 76, 1007 (1996); Phys. Rev. D 54, 1332 (1996).

[25] M. Kamionkowski, A. Kosowsky, and A. Stebbins, Phys. Rev. D 55, 7368 (1997); M. Zaldarriaga and U. Seljak, Phys. Rev. D 55, 1830 (1997).

[26] E. Ripamonti, M. Mapelli, and A. Ferrara, Mon. Not. R. Astron. Soc. 375, 1399 (2007); 374, 1067 (2007). 